\title{
Interference Empowered 5G Networks
}

\author{
Matthieu de Mari \\ Zdenek Becvar \\ Emilio Calvanese Strinati \\ Merouane Debbah \\ matthieu.demari@supelec.fr zdenek.becvar@fel.cvut.cz \\ emilio.strinati@cea.fr \\ merouane.debbah@supelec.fr
}

\begin{abstract}
In future 5G networks, in-band interference is perceived as one of the most critical performance bottlenecks. While current solutions classically treat interference as an additional source of noise, recent advances in information theory show that interference is not necessarily an opponent, but might be cancelled or supressed. In this paper, we propose a threefold optimization method, which couples reduced complexity interference classification and matching techniques, to enhance the system performance. The matching objective consists in i) defining coalitions of users assigned to each Access Points (AP); ii) match interferers that will be transmitting on the same spectral resources into groups of interferers; and iii) define the transmission rates and interference regimes for each user inside each group. Our analytical study and simulations results show that our proposed solution allows for system spectral efficiency enhancements, compared to classical reference scenarios.
\end{abstract}

\section{INTRODUCTION}

In this paper, we address the everlasting problem of interference that strongly affects the network performance: in-band interference. Common understanding is that interference compromises the reliability of transmissions and therefore must be strongly limited or avoided. In that sense, several Radio Resource Management (RRM) schemes are designed to limit the undesired effects of interference. However spectrally suboptimal, partial or full orthogonalization, via time sharing or frequency orthogonalization, allows interference to be avoided or at least strongly limited [1]. More efficient techniques, such as power balancing techniques, involve more mathematical complexity and might become intractable when the number of users in the system grows large [2][3][4]. Recent advances in information theory have also shown that interference does not necessarily have to be avoided or strongly limited, especially in cases where interference might be strong enough to be decoded and canceled out of the received signal [5]. In a detailed theoretical study, Etkin and Tse [6] have defined a 5 interference regimes classification, i.e. the 5 most appropriate ways to process interference at the receiver side, for any given interference to signal ratio, as well as the spectral efficiency that the receiver can benefit from each technique after interference processing. This classification was later simplified by Abgrall [7], into a 3 regimes classification. Based on this observation, self-organizing networks, capable of interference suppression or cancellation were proposed as a possible key enhancement for 3GPP LTE and 5G future networks [8][9]. In a previous paper [10], we exploited this interference classification and investigated an optimization problem where a couple of interferers could cooperate and adapt their transmission rates and interference perceptions, so that interference could either be treated as noise or decoded and canceled out via
Successive Interference Cancellation based techniques. Later on, in [11], we extended the problem and considered a scenario with $M=2$ Access Points (APs) and $N$ User Equipments (UEs) assigned to each AP and investigated the matching problem consisting of forming groups of interferers, sharing common spectral resources, assuming the interferers would implement interference regimes according to [10]. The optimal matching was then the one that maximized the total spectral efficiency of the system. This two-steps optimization led to notable performance improvements, in terms of total spectral efficiency, compared to reference scenarios where the matching was random, interference necessarily treated as noise, and/or interference was avoided via orthogonalization.

However, in the two previous papers, we assumed that the AP-UE assignments were pre-established: this assumption strongly limits the optimization space of interference aware RRM mechanisms. It also appears that when two users can treat interference using SIC-based techniques, system's performance is improved by transmitting using the interfering links, instead of its pre-assigned ones (as shown in Proposition III.2). For this reason, it makes sense to provide an additional degree of freedom to the system, by letting the system change its AP-UE assignments, instead of arbitrarily fixing them. In this paper, we present a technique that allows to recompile, at each allocation instance, the matchup between AP and users. This additional assignment procedure complexifies the optimization procedure. We now consider in this paper, a threefold optimization problem where the system has to i) define equal coalitions of UEs assigned to each AP; ii) identify groups of interferers, with one interferer from each coalition, that will transmit over the same spectral resources and interfere; and iii) define the most appropriate transmission rates and interference regimes to be used by each UE in each group of interferers, according to our interference classifier. The objective of the conducted optimization is to maximize the total spectral efficiency of the system.

In section II, we define the system model, the optimization problem to be investigated throughout the paper and detail a short reminder of our previous interference classification works. In section III, we update our previous interference classifier [10] and take into account the possibility offered to the system to change its AP-UE assignments. This leads to a "2-Regime Interference Classifier". In section IV, we detail the new matching problem to be considered in the 2 APs and multiple unassigned UEs scenario, and investigate from the graph theory point of view, a method to solve the optimization problem. In section $\mathrm{V}$, we take a glance at the 
matching problem detailed in the previous section, when the number of APs is greater than 2, with multiple unassigned UEs. Finally, we provide numerical simulations that assess the notable performance improvements, in terms of total spectral efficiency, compared to state-of-the-art reference scenarios.

\section{SYSTEM MODEL AND OPTIMIZATION PROBLEM}

We consider a system, consisting of $M$ APs and $N_{u}=M N$ UEs (i.e. $N$ UEs/AP). For any $i \in\left\{1, \ldots, N_{u}\right\}, k \in\{1, \ldots, M\}$, we denote $h(k, i)$ the channel between AP $k$ and UE $i$. If the AP $k$ is used for transmission to UE $i$, the combination AP $k$ and UE $i$ can be referred to as "interferer $(k, i)$ ". We denote $p(k, i)$ the transmission power from AP $k$ to UE $i$. For a given disjoint combination of UEs $\left(i_{1}, i_{2}, \ldots i_{M}\right) \in\left\{1, \ldots, N_{u}\right\}^{M}$, we assume that the transmission powers are fixed and that $\forall k \in$ $\{1, \ldots, M\}, \forall i \in\left(i_{1}, i_{2}, \ldots i_{M}\right), p(k, i)=p_{k}\left(i_{1}, i_{2}, \ldots i_{M}\right)>0$. Finally, the noise is assumed to be Gaussian, with variance $\sigma_{n}^{2}$. We also assume that each UE in $\left(i_{1}, i_{2}, \ldots i_{M}\right)$ is assigned to one and only one of the $M$ AP. This assumption allows for a simple mathematical problem with low complexity, and we showed in [11] that it helps the matching process detailed hereafter converge to a solution.

\section{A. State-of-the-Art and new Interference Classification Regimes}

We first focus on the case $M=2, N=1$. As in [10][11], we also assume that the UEs-APs assignments are pre-established, i.e. $\forall i \in\{1,2\}$, UE $i$ is assigned to AP $i$. Also, the two interferers share the same spectral resources and suffer from interference. Downlink transmissions occur from each AP $i$ to its assigned UE $i$ and an interference channel can be considered, as depicted in Figure 1.

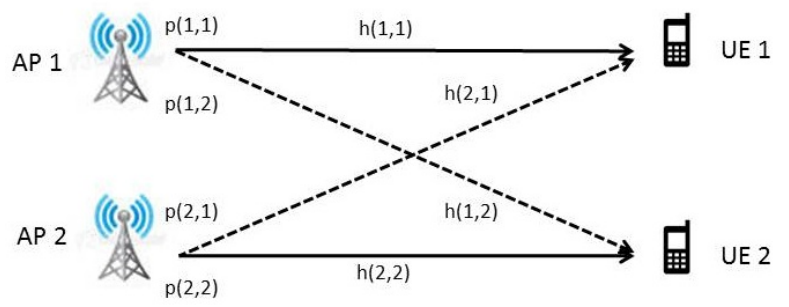

Fig. 1. A simple interference channel when $M=2$.

We also denote $\Gamma$ the set of all signal to noise ratios $\gamma(i, j)$ related to the two interferers:

$$
\begin{array}{r}
\forall(i, j) \in\{1,2\}^{2}, \gamma(i, j)=\frac{p(j, i)|h(j, i)|^{2}}{\sigma_{n}^{2}} \\
\Gamma=(\gamma(1,1), \gamma(2,2), \gamma(1,2), \gamma(2,1))
\end{array}
$$

As in [10][11], we assume that the APs cooperate, that perfect knowledge of the transmission settings are given to the APs and that each interferer $(i, i)$ is able to process interference according to 3 possible regimes. We denote $O_{i}$ the interference regime of interferer $i$. As defined in [7], the

\begin{tabular}{|c|c|}
\hline $\mathcal{O}$ & $R(\mathcal{O}, \Gamma)=R_{1}(\mathcal{O}, \Gamma)+R_{2}(\mathcal{O}, \Gamma)$ \\
\hline$(1,1)$ & $\log _{2}\left(1+\frac{\gamma(1,1)}{1+\gamma(2,1)}\right)+\log _{2}\left(1+\frac{\gamma(2,2)}{1+\gamma(1,2)}\right)$ \\
\hline$(2,2)$ & $\frac{1}{2} \log _{2}(1+\gamma(1,1))+\frac{1}{2} \log _{2}(1+\gamma(2,2))$ \\
\hline$(3,1)$ & $\log _{2}(1+\gamma(1,1))+\log _{2}\left(1+\min \left[\frac{\gamma(2,2)}{1+\gamma(1,2)}, \frac{\gamma(2,1)}{1+\gamma(1,1)}\right]\right)$ \\
\hline$(1,3)$ & $\log _{2}\left(1+\min \left[\frac{\gamma(1,1)}{1+\gamma(2,1)}, \frac{\gamma(1,2)}{1+\gamma(2,2)}\right]\right)+\log _{2}(1+\gamma(2,2))$ \\
\hline$(3,3)$ & $\begin{array}{l}\log _{2}\left(1+\min \left[\gamma(1,1), \frac{\gamma(1,2)}{1+\gamma(2,2)}\right]\right) \\
+\log _{2}\left(1+\min \left[\gamma(2,2), \frac{\gamma(2,1)}{1+\gamma(1,1)}\right]\right)\end{array}$ \\
\hline$(1,1)^{*}$ & $\log _{2}\left(1+\frac{\gamma(2,1)}{1+\gamma(1,1)}\right)+\log _{2}\left(1+\frac{\gamma(1,2)}{1+\gamma(2,2)}\right)$ \\
\hline$(2,2)^{*}$ & $\frac{1}{2} \log _{2}(1+\gamma(2,1))+\frac{1}{2} \log _{2}(1+\gamma(1,2))$ \\
\hline$(3,1)^{*}$ & $\log _{2}(1+\gamma(2,1))+\log _{2}\left(1+\min \left[\frac{\gamma(1,1)}{1+\gamma(2,1)}, \frac{\gamma(1,2)}{1+\gamma(2,2)}\right]\right)$ \\
\hline$(1,3)^{*}$ & $\log _{2}\left(1+\min \left[\frac{\gamma(2,2)}{1+\gamma(1,2)}, \frac{\gamma(2,1)}{1+\gamma(1,1)}\right]\right)+\log _{2}(1+\gamma(1,2))$ \\
\hline$(3,3)^{*}$ & $\begin{array}{l}\log _{2}\left(1+\min \left[\gamma(2,1), \frac{\gamma(2,2)}{1+\gamma(1,2)}\right]\right) \\
+\log _{2}\left(1+\min \left[\gamma(1,2), \frac{\gamma(1,1)}{1+\gamma(2,1)}\right]\right)\end{array}$ \\
\hline$(2,2)^{1}$ & $\frac{1}{2} \log _{2}(1+\gamma(1,1))+\frac{1}{2} \log _{2}(1+\gamma(1,2))$ \\
\hline$(2,2)^{2}$ & $\frac{1}{2} \log _{2}(1+\gamma(2,1))+\frac{1}{2} \log _{2}(1+\gamma(2,2))$ \\
\hline
\end{tabular}
3 regimes are:
CONFIGURATIONS $\mathcal{O}$ AND THEIR SPECTRAL EFFICIENCIES PERFORMANCES: 5 FIRST REGIMES FROM [10][11] AND 7 NEW REGIMES

Noisy $-O_{i}=1$ : the interference is weak enough to be processed as additive noise at the receiver side.

Successive Interference Cancellation (SIC) - $O_{i}=3$ : UE $i$ can decode the strong incoming interference and cancel it out of the received signal using SIC.

Orthogonalization $-O_{i}=2$ : interferer $(i, i)$ attempts to avoid interference by transmitting using only the $i$ th half of spectral resources. If interferer $(j, j)$, with $j \neq i$, performs orthogonalization as well, interference is avoided, at the cost of using only half of the spectral resources.

In this section, we present in in Table I the interference regimes that were initially proposed in [10][11] and new sets of interference regimes that extend our previous regimes to cases in which changing the AP-UE assignments is permitted. For any given realization of $\Gamma$, we defined in [10][11] the rates $R_{i}$ at which each interferer $i$ can transmit without outage occurring. We also provided criteria that immediately states the interference regimes perform the best, i.e. returns the best total spectral efficiency $R(\mathcal{O}, \Gamma)=\sum_{i} R_{i}$. It also appeared that among the 9 possible combinations for $\mathcal{O}=\left(O_{1}, O_{2}\right)$, there were only 4 regimes of interest for our couple of interferers, namely $(1,1),(3,1),(1,3)$ and $(3,3)$, whose performance are given in Table I. We now assume that the system is allowed to modify the initial UEs-APs assignments, i.e. UE $i$ can be assigned to $\operatorname{AP} j(j \neq i)$, instead of $\mathrm{AP} i$, as we 
supposed before. We might then consider new regimes, that take into account the APs-UEs assignments, thus offering new possibilities of assignments. In the following, we denote:

- $\mathcal{O}=\left(O_{1}, O_{2}\right)$ : this refers to the configuration where UE 1 (resp. UE 2) is assigned to AP 1 (resp. AP 2) and UE 1 (resp. UE 2) treats interference coming from AP 2 (resp. AP 1) according to $O_{1}$ (resp. $O_{2}$ ).

- while $\mathcal{O}=\left(O_{1}, O_{2}\right)^{*}$ : this refers to the exact same thing, except UE 1 (resp. UE 2) is associated to AP 2 (resp. AP $1)$.

We also define $\forall i \in\{1,2\},(2,2)^{i}$ the configuration where both UEs are assigned to the same AP $i$ and perform equal orthogonalization, in order to avoid interference. It leads to 12 admissible configurations, whose spectral efficiencies performances are listed in Table I. $R_{1}$ (resp. $R_{2}$ ) refers to the spectral efficiency of UE 1 (resp. UE 2), whereas $R=R_{1}+R_{2}$ is the global spectral efficiency of the couple of interferers.

In this paper, we first focus on defining, for a given $\Gamma$, the best UE-AP associations and interference regimes, i.e the best configuration $\mathcal{O}$, such that the total spectral efficiency $R(\mathcal{O}, \Gamma)$ of the system is maximized. There are 12 possible configurations $\mathcal{O}$ listed in Table I, the first five configurations were already detailed in [10].

\section{INTERFERENCE CLASSIFICATION AND ASSIGNMENTS, WITH $N_{u}=2$ INTERFERERS}

In this paper, and as in [10][11], we define the $\triangleright$ operator, where $\mathcal{O} \triangleright \mathcal{O}^{\prime}$ means that the configuration $\mathcal{O}$ offers a better maximal total spectral efficiency $R$ than $\mathcal{O}^{\prime}$ :

$$
R(\mathcal{O}, \Gamma) \geq R\left(\mathcal{O}^{\prime}, \Gamma\right)
$$

Our objective in this section is twofold: i) we wish to identify configurations of interest, i.e. configurations that can potentially be the best performing configurations for certain realizations of $\Gamma$; ii) we define criteria on $\Gamma$ that immediately tell which configuration of interest is the best performing configuration. Let us now consider the two following propositions that allow for simplifications.

Proposition III.1. For any given channel realization $\Gamma$ and any configuration inducing orthogonalization on both sides, there exists a configuration that outperforms it. More precisely:

- $(2,2)$ is outperformed by either $(1,3),(3,1)$.

- $(2,2)^{*}$ is outperformed by either $(1,3)^{*},(3,1)^{*}$.

- $(2,2)^{1}$ is outperformed by either $(2,3)^{*},(3,2)$.

- $(2,2)^{2}$ is outperformed by either $(3,2)^{*},(2,3)$.

Note that $(2,3)$ and $(3,2)$ were regimes defined in [10][11]. Details about these configurations are given in proof.

Proof. Refer to [12], Section I.

Proposition III.2. In scenarios, where both users can decode and cancel interference using SIC-based techniques, it is more interesting for the system to transmit using the interfering links, instead of its pre-assigned ones and treat interference as noise, i.e., $\forall \Gamma$ :
- $(3,3) \triangleright(1,1)^{*}$.

- $(3,3)^{*} \triangleright(1,1)$.

Proof. Refer to [12], Section II.

Based on the previous propositions, we have shown that our classifier only operates within 6 configurations of interest, namely $(1,1),(1,3),(3,1),(1,1)^{*},(1,3)^{*}$ and $(3,1)^{*}$. Also, no orthogonalization-based configuration subsists, as they are all outperformed by at least one of the 6 configurations of interest. As in the previous "2-Regimes Interference Classifier" [10][11], each interferer can only treat interference according to 2 interference regimes: $O_{i}=1$ (Noisy) or 3 (SIC). Moreover, the UEs-APs assignments of each configuration guarantee that each AP is assigned one and only one UE, meaning that no beamforming is yet assumed.

We define the best performing configuration (BPC) as follows: $\forall \Gamma \in \Omega, \mathcal{O}^{\prime}$ is the best performing configuration (BPC) in region $\Omega$, if and only if there does not exist any configuration $\mathcal{O}$ that outperforms $\mathcal{O}^{\prime}$, i.e.:

$$
\forall \mathcal{O}, \forall \Gamma \in \Omega, R\left(\mathcal{O}^{\prime}, \Gamma\right) \geq R(\mathcal{O}, \Gamma)
$$

We now focus on defining regions $\Omega$, where each configuration of interest is the best interference configuration. Our "6-Regimes Configuration Classifier" defined in Proposition III.3 returns, for any channel configuration $\Gamma$, the AP-UE assignment and the interference regimes, corresponding to the best performing configuration.

Proposition III.3. We define the "6-Regimes Configuration Classifier", as follows.

1) If $\gamma(1,1) \geq \gamma(1,2)$ and $\gamma(2,2) \geq \gamma(2,1)$

- $(1,1) B P C \Leftrightarrow \gamma(1,1) \geq \gamma(1,2)(1+\gamma(2,1))$ and $\gamma(2,2) \geq \gamma(2,1)(1+\gamma(1,2))$

- $(1,3) B P C \Leftrightarrow(1,1)$ not BPC and $\gamma(2,2)+\gamma(1,2) \geq$ $\gamma(1,1)+\gamma(2,1)$

- $(3,1) B P C \Leftrightarrow(1,1)$ not BPC and $\gamma(2,2)+\gamma(1,2) \leq$ $\gamma(1,1)+\gamma(2,1)$

2) If $\gamma(1,1) \leq \gamma(1,2)$ and $\gamma(2,2) \leq \gamma(2,1)$

- $(1,1)^{*} B P C \Leftrightarrow \gamma(2,1) \geq \gamma(2,2)(1+\gamma(1,1))$ and $\gamma(1,2) \geq \gamma(1,1)(1+\gamma(2,2))$

- $(1,3)^{*} B P C \Leftrightarrow(1,1)^{*}$ not $B P C$ and $\gamma(2,2)+\gamma(1,2) \geq$ $\gamma(1,1)+\gamma(2,1)$

- $(3,1)^{*} B P C \Leftrightarrow(1,1)^{*}$ not $B P C$ and $\gamma(2,2)+\gamma(1,2) \leq$ $\gamma(1,1)+\gamma(2,1)$

3) If $\gamma(1,1) \geq \gamma(1,2)$ and $\gamma(2,2) \leq \gamma(2,1)$

- $(3,1) B P C \Leftrightarrow \frac{\gamma(1,1)}{1+\gamma(2,1)} \geq \frac{\gamma(1,2)}{1+\gamma(2,2)}$ and $(1+\gamma(1,1))(1+\gamma(2,2) \geq(1+\gamma(2,1))(1+\gamma(1,2))$

- $(3,1)^{*} B P C \Leftrightarrow \frac{\gamma(2,1)}{1+\gamma(1,1)} \geq \frac{\gamma(2,2)}{1+\gamma(1,2)}$ and

$$
(1+\gamma(1,1))(1+\gamma(2,2) \leq(1+\gamma(2,1))(1+\gamma(1,2))
$$

4) If $\gamma(1,1) \leq \gamma(1,2)$ and $\gamma(2,2) \geq \gamma(2,1)$

- $(1,3)^{*} B P C \Leftrightarrow \frac{\gamma(1,1)}{1+\gamma(2,1)} \leq \frac{\gamma(1,2)}{1+\gamma(2,2)}$ and

$$
(1+\gamma(1,1))(1+\gamma(2,2) \leq(1+\gamma(2,1))(1+\gamma(1,2))
$$




$$
\begin{aligned}
-(1,3) B P C \Leftrightarrow \frac{\gamma(2,1)}{1+\gamma(1,1)} \leq \frac{\gamma(2,2)}{1+\gamma(1,2)} \text { and } \\
\quad(1+\gamma(1,1))(1+\gamma(2,2) \geq(1+\gamma(2,1))(1+\gamma(1,2))
\end{aligned}
$$

Proof. Extended and detailed proof of this proposition is available online, in [12], Section III.

We have then defined an updated version of our previous "2-Regime Interference Classifier", that takes into account both the interference regimes and the AP-UE assignments for couples of interferers.

\section{Optimization, Assignment And Matching}

\section{A. Matching Problem, with $M=2, N>1$}

In this section, we consider the matching problem with $M=2$ APs. $N_{u}=2 N$ unassigned UEs must be first divided in two coalitions of $N$ UEs (i.e. the AP-UE assignments for both APs must be defined). Assuming we have split the available resources in $N$ equal elements, one UE from each coalition must then be assigned to each spectral resource. The interferers sharing a same spectral resource are assigned to different APs and suffer from interference. This leads to a twofold optimization problem, where the objective is to maximize the total spectral efficiency of the system i) by forming pairs of interferers and ii) by defining AP-UE assignments, as well as interference regimes, i.e. the best configurations.

The problem is addressed in a two-steps optimization. First, for any pair of interferers, our previous configuration classifier gives the best configuration to be used. The second step consists of finding the $N$ disjoint pairs of interferers, i.e. the interferers assignments $m^{*}$, that maximize the total spectral efficiency $\mathcal{R}$

$$
m^{*}=\arg \max _{m}\left[\mathcal{R}=\frac{1}{2} \sum_{i=1}^{N_{u}} R^{\prime}(i, m(i), \Gamma(i, m(i))]\right.
$$

Where

- $\forall i_{1}, i_{2} \in\left\{1, \ldots, N_{u}\right\}, i_{1} \neq i_{2}, m\left(i_{1}\right)=i_{2}$ means that interferer $i_{1}$ is coupled with interferer $i_{2}$. If $m\left(i_{1}\right)=i_{2}$, then necessarily, $m\left(i_{2}\right)=i_{1}$. This is sufficient to guarantee a disjoint matching of interferers.

- $\Gamma\left(i_{1}, i_{2}\right)$ plays the same role as $\Gamma$ in Section III and contains the $\gamma$ elements related to UEs $i_{1}$ and $i_{2}$.

The maximal spectral efficiency $R^{\prime}\left(i_{1}, m\left(i_{1}\right), \Gamma\left(i_{1}, m\left(i_{1}\right)\right)\right.$ that UEs $i_{1}$ and $m\left(i_{1}\right)$ can pretend to is given by our previous configuration classifier. The classifier returns the optimal APUE assignment, the interference regimes for our pair of interferers, and the maximal spectral performances for our couple of interferers. Assuming we can define the optimal configuration and compute their maximal joint spectral efficiency $R^{\prime}(i, j, \Gamma(i, j)), \forall(i, j) \in\left\{1, \ldots, N_{u}\right\}, i \neq j$, our objective is now strictly equivalent to finding the $N$ disjoint pairs of interferers (or the assignments $m^{*}$ ) that maximize the total spectral efficiency $\mathcal{R}$. From the graph theory point of view, this consists of finding a maximum weight disjoint edges matching, i.e. select $N$ edges, with no two edges sharing a same node, such that the sum of edges is maximized. We address this problem in the case of a $N_{u}$-nodes complete graph, each node referring to an interferer. The weights between two nodes $i$ and $j$ is then given by $R(i, j, \Gamma(i, j))$. This matching problem is strictly equivalent to the previous optimization problem (3), which is easily solved by a combinatorial algorithm using a weighted Edmonds's algorithm [13][14].

\section{B. Numerical Simulations and Performance Insights}

In order to highlight the performance gains of our optimization approach, we run Monte-Carlo simulations, with $N_{M C}=1000$ independent realizations. We have considered two APs, within a distance of $d_{A P}$. The $N_{u}=2 N$ unassigned UEs are uniformly distributed in the coverage area of each AP $R_{A P}$. In the following, we denote $d(i, j)$ the distance between $\mathrm{AP} i$ and $\mathrm{UE} j$. The channels $h(i, j)$ include the antenna gain $G$, the path loss $L(d(i, j))$ and the shadowing $\xi$. All parameters are summarized in Table II, and based on [15].

\begin{tabular}{|l|l|}
\hline Parameter & Value \\
\hline \hline Distance between AP to AP $d_{A P}$ & $1 \mathrm{~km}$ \\
\hline Coverage Area $R_{A P}$ & $\begin{array}{l}\text { Users are unif. dist. } \\
\text { s.t. dist. AP-UE } \in\left[r_{\min }, r_{m a x}\right]\end{array}$ \\
\hline$\left[r_{\min }, r_{\max }\right]$ & {$[35 m, 750 \mathrm{~m}]$} \\
\hline Transmission powers $p_{k}()$. & Proportional to dist. $\in[20 \mathrm{dBm}, 46 \mathrm{dBm}]$ \\
\hline Channels $h(i, j)$ & $h(i, j)=\frac{G}{L(d(i, j)) \xi}$ \\
\hline Antenna Gain $G$ & $10 \mathrm{dBi}$ \\
\hline Path Loss $L(d(i, j)),[d$ in $\mathrm{km}]$ & $L=131.1+42.8 \log _{10}(d(i, j))$ \\
\hline Shadowing $\xi$ & Log-normal, $\sigma_{S H}=10 \mathrm{~dB}$ \\
\hline Noise power $\sigma_{n}$ & $-104 \mathrm{dBm}$ \\
\hline Number of unassigned UEs $N_{u}$ & 50 \\
\hline \multicolumn{2}{|c|}{ TABLE II } \\
\hline
\end{tabular}

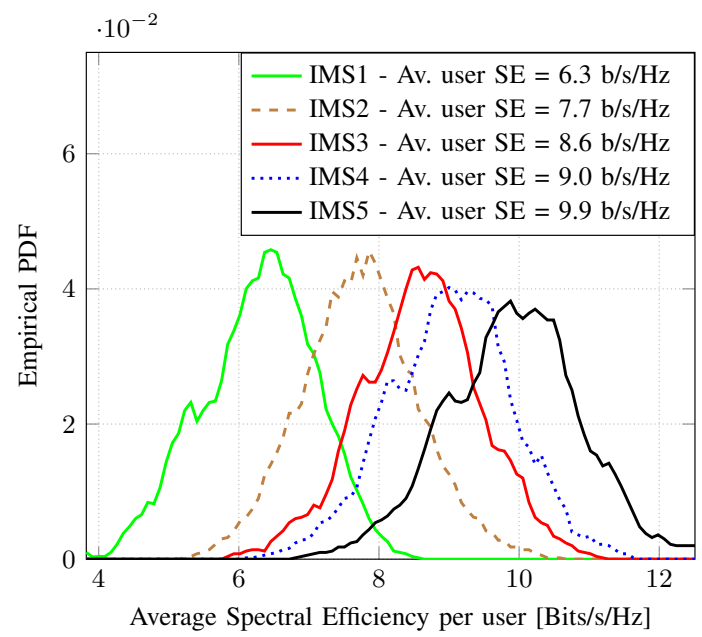

Fig. 2. Fitted histogram plot of the performances of each scenario, for $N_{M C}=1000$ independent realizations ( 2 interferers per group).

We consider 5 interference management strategies (IMS) of interest:

IMS 1: orthogonalization is performed so that no UEs are interfering and each UE is assigned to its best AP. 
IMS 2: UEs-APs assignments are randomly defined, so that each AP has $N$ assigned UEs, but prioritize the UEs closest to the APs. More details on this random assignment procedure can be found in [11]. Interferers matching is also random. Interferers have no other choice but to treat incoming interference as an additive source of noise.

IMS 3: UEs-APs assignments are randomly defined, so that each AP has $N$ assigned UEs, but prioritize the UEs closest to the APs. More details on this random assignment procedure can be found in [11]. Interferers matching is also random. Interferers can treat interference according to the best admissible interference regime.

IMS 4: UEs-APs assignments are randomly defined, so that each AP has $N$ assigned UEs, but prioritize the UEs closest to the APs. More details on this random assignment procedure can be found in [11]. Best interferers matching is computed with Kuhn-Munkres Algorithm [16]. Interferers can treat interference according to the best admissible interference regime.

IMS 5: Define the optimal configuration with optimal UEs-APs assignments, interferers matching and interference regimes.

We present in Figure 2, the distribution of the spectral efficiency per user and the average performance in terms of spectral efficiency per user, for each IMS, over $N_{M C}=1000$ independent Monte-Carlo simulations. We observe that full orthogonalization, i.e. IMS 1 , is spectrally inefficient. This is an expected result that we have already shown in [11]. We now consider as a reference IMS 2, where the assignments and matchings are both random, and interference is treated as additive noise. Optimizing the interference regimes, the interferers matchings and the AP-UE assignments lead to notable performance improvements. More precisely, allowing the system to select the best interference regime (IMS 3) offers an average performance improvement of $11.1 \%$, compared to $I M S$ 2. Furthermore, allowing the system to select the most appropriate interferers matching (IMS 4), leads to an average performance improvement of $16.8 \%$. Finally, allowing the system to select the most appropriate UEs-APs assignments (IMS 5) allows an summed up average performance improvement of $28.0 \%$, compared to IMS 2 .

The overall gain between IMS 2 and IMS 5 can be decomposed in 3 parts:

Interference Classification Gain: First, the gain offered by interference classification (between IMS 2 and IMS 3) depends on the proportion of interferers implementing a SIC regime, instead of a noisy one.

Interferers Matching Gain: Secondly, there is a gain related to the matching of interferers (between IMS 3 and IMS 4), which seems to depend on the variance of the signal to noise ratios $\gamma(i, j)$. In fact, it is easy to picture that there would be no gain between the best matching and the random matching if the INRs/ SNRs $\gamma(i, j)$ were the same for every interferer.
AP-UE Assignments Gain: Finally, there also appear to be a notable gain, related to the capability offered to the system to assign the UEs to a more appropriate AP, and not necessarily the one providing the best SNR (between $I M S 4$ and IMS 5). This gain also seems to scale with the diversity of AP offered to any interferer of the system, but it needs further investigation.

On this specific topic, exhaustive benchmark is still on going.

\section{EXTENSION TO LARGE NUMBER OF INTERFERERS AND LIMITS}

\section{A. Interference Classification with more than 2 interferers}

In section III, we defined an interference and assignment classifier when the number of interferers was $M=2$. When the number of interferers to be matched together becomes larger than 2, defining interference regimes may involve Joint Decoding or $k$-SIC techniques, for which it appears complicated to define spectral performances [17]. Also, the number of sub-cases grows extremely fast with the number of interferers in a group $M$. Nevertheless, in this paper, we carry on with the matching problem, by assuming that the interference is always treated as an additive source of noise, at each receiver side. In such a configuration, we are still able to highlight the performance gains of our matching approach, and the potential benefit that appears from being able to smartly define groups of interferers and AP-UE assignments.

\section{B. Matching Problem, when $M>2, N>1$}

The objective now consists of finding the matchings $m$ and $u$, where $\forall i \in\{1, \ldots, M\}, \forall j \in\left\{1, \ldots, N_{u}\right\}, \forall k \in\{1, \ldots, N\}$ :

$$
m(i, j)= \begin{cases}1 & \text { if } \mathrm{UE} \mathrm{j} \text { is assigned to AP } \mathrm{i} \\ 0 & \text { else }\end{cases}
$$

$$
u(k, j)= \begin{cases}1 & \text { if } \mathrm{UE} \mathrm{j} \text { is assigned to group of interf. } \mathrm{k} \\ 0 & \text { else }\end{cases}
$$

The matchings are constrained: each UE is assigned to one and only one AP and group of interferers, i.e. $\forall j \in$ $\left\{1, \ldots, N_{u}\right\}$ :

$$
\sum_{i=1}^{M} m(i, j)=1 \text { and } \sum_{k=1}^{N} u(k, j)=1
$$

In section IV-A, we showed that the matching problem with $M=2$ consisted of a maximum disjoint edges weighted matching in a complete $N_{u}$ graph. If we now assume that $M>2$, the objective is more complicated and can not be expressed from a graph theory point of view. The only approach that exists, to the best of our knowledge, consists of Integer Linear Programming, which is known to be NP-Hard [18]. Nevertheless, in small dimensions systems (i.e. for small values of $N$ and $M$ ), branch-and-bound algorithms are able to return optimal matchings in acceptable computation times. 


\section{Numerical Simulations}

In this section, we assume that the simulations parameters will be the same as those listed in section IV-B. We consider a system with 5 Macro-APs and 5 Femto-APs ( $M=10$ APs) and $N_{u}=N M=50$ UEs. We consider reference IMS 1) and 2 ), defined previously, as well as IMS 3b), defined hereafter:

IMS 1: orthogonalization is performed so that no UEs are interfering and each UE is assigned to its best AP.

IMS 2: UEs-APs assignments are randomly defined, so that each AP has $N$ assigned UEs, but prioritize the UEs closest to the APs. Interferers matching is also random. Interferers have no other choice but to treat incoming interference as an additive source of noise.

IMS 3: Best optimization scenario with optimal UEs-APs assignments, interferers matching. Interference treated as an additive source of noise at each receiver side.

We present in Figure 3 the histogram of the average spectral efficiency per UE, for each IMS.

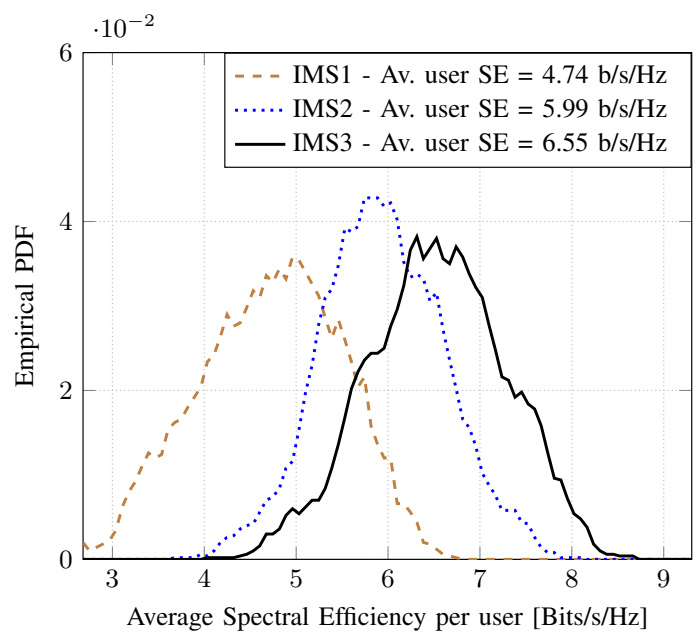

Fig. 3. Fitted histogram of the performances of each scenario, for $N_{M C}=$ 1000 independent realizations ( $M>2$ interferers per coalition).

As in section IV-B, it appears that IMS 1, is spectrally inefficient. Considering IMS 2 as a reference, it appears that optimizing the interferers matchings and the AP-UE assignments lead to notable performance improvements: allowing the system to select the best matching of interferers in the noisy regime (IMS 3) offers an average performance improvement of $9.9 \%$, compared to IMS 2. Gains remain limited compared to section IV-B, as we are unable to implement interference classification in multiple interferers situations.

\section{CONCLUSION}

$5 \mathrm{G}$ networks will be deployed much more densely, heterogeneously and with different degrees of centralized processing. Interference is still perceived as a major bottleneck for meeting theoretical and on-field performance. In this paper, we propose a novel interference handling approach which implement interference classification. To this end, we investigate the optimization problem which aims at adapting the interference regimes of a given set of UE, forming groups of interferers transmitting over the same spectral resources, as well as defining the AP-UE assignments. For the $M=2$ APs case, an optimal procedure is proposed. For larger systems $(M>2)$, we have shown that the problem rapidly becomes NP-Hard and proposed an approach based on Integer Linear Programming. By numerical simulations means, we showed that our proposed solution leads to notable performance improvements compared to classical RRM procedures. Future work will investigate interference regimes with $M>2$ interferers which may involve Joint Decoding or $k$-SIC techniques, as well as heuristic algorithms for solving the matching in large networks scenarios.

\section{REFERENCES}

[1] D. Gesbert, S. G. Kiani, A. Gjendemsjo, et al., "Adaptation, coordination, and distributed resource allocation in interference-limited wireless networks," Proceedings of the IEEE, vol. 95, no. 12, 2007.

[2] O. Sahin, E. Erkip, and D. Goodman, "Iterative power control for multimedia wireless communications," in Acoustics, Speech and Signal Processing, 2006. ICASSP 2006 Proceedings. 2006 IEEE International Conference on, vol. 4, pp. IV-IV, IEEE, 2006.

[3] T. Holliday, A. Goldsmith, N. Bambos, and P. Glynn, "Distributed power and admission control for time-varying wireless networks," in Information Theory, 2004. ISIT 2004. Proceedings. International Symposium on, pp. 352-352, IEEE, 2004.

[4] C. Abgrall, E. C. Strinati, and J.-C. Belfiore, "Distributed power allocation for interference limited networks," in Personal Indoor and Mobile Radio Communications (PIMRC), 2010 IEEE 21st International Symposium on, pp. 1342-1347, IEEE, 2010.

[5] A. Carleial, "A case where interference does not reduce capacity (corresp.)," Information Theory, IEEE Transactions on, vol. 21, pp. 569570, Sep 1975.

[6] R. H. Etkin, D. N. Tse, and H. Wang, "Gaussian interference channel capacity to within one bit," Information Theory, IEEE Transactions on, vol. 54, no. 12, pp. 5534-5562, 2008.

[7] C. Abgrall, Allocation de ressources dans les réseaux sans fil denses. PhD thesis, Télécom ParisTech, 2010.

[8] “3gpp tr36.829 v11.1.0 "enhanced performance requirement for lte user equipment (ue)"," 2013.

[9] "3gpp tr 36.866 v12.0.1 "study on network-assisted interference cancellation and supression"," 2014.

[10] M. De Mari, E. C. Strinati, and M. Debbah, "Two-regimes interference classifier: an interference-aware resource allocation algorithm," in Wireless Communications and Networking Conference, 2014. IEEE WCNC 2014. IEEE, IEEE, 2014.

[11] M. De Mari, E. C. Strinati, and M. Debbah, "Interference classification and matching: Two steps optimization for improving spectral efficiency," in PIMRC, 2014. Available here: http://matthieu-de-mari.fr/wp-content/ uploads/2014/07/IEEETransWC_2014_1_Journal_draft.pdf.

[12] M. De Mari, E. C. Strinati, and M. Debbah, Elements of Proof: Interference Empowered $5 G$ Networks. Available here: http://matthieu-de-mari. fr/wp-content/uploads/2014/09/5GU_2015_EOP_final.pdf.

[13] L. Lovász and M. D. Plummer, Matching Theory. 1986.

[14] V. Kolmogorov, "Blossom v: a new implementation of a minimum cost perfect matching algorithm," Mathematical Programming Computation, vol. 1, no. 1, pp. 43-67, 2009.

[15] G. T. RAN, "“"tr 36.872, small cells enhancements for e-utra and e-utran - physical layer aspects (release 12), v12.1.0, december 2013,"

[16] H. W. Kuhn, "The hungarian method for the assignment problem," Naval research logistics quarterly, vol. 2, no. 1-2, pp. 83-97, 1955.

[17] J. Blomer and N. Jindal, "Transmission capacity of wireless ad hoc networks: Successive interference cancellation vs. joint detection," in Communications, 2009. ICC'09. IEEE International Conference on, pp. 1-5, IEEE, 2009.

[18] G. L. Nemhauser and L. A. Wolsey, Integer and combinatorial optimization, vol. 18. Wiley New York, 1988. 